\title{
Multiplexing Techniques of Single Flux Quantum Circuit Based Readout Circuit for a Multi-Channel Sensing System
}

\author{
K. Aoki, Y. Yamanashi, and N. Yoshikawa, Member, IEEE
}

\begin{abstract}
Time division multiplexing (TDM) and code division multiplexing (CDM) have been investigated for multi-channel superconductive sensing systems using single-flux-quantum (SFQ) readout circuits. Output data from a superconductive sensor array can be multiplexed using SFQ binary counters, which count the number of SFQ pulses from each sensor, and are transmitted from a low-temperature environment to roomtemperature equipment using a small number of lines. We have estimated and compared the performance of a multi-channel superconductive sensing system that employs TDM and CDM on the basis of analog circuit simulation and circuit design results. TDM is useful for reducing the number of lines, but the slew rate of the sensing system decreases with an increase in the number of channels. On the other hand, in the case of CDM, the slew rate of the system does not decrease with an increase in the number of channels. We have designed and tested a 2-channel digital SQUID system that can perform TDM and CDM employing SFQ up/down binary counters. In both circuits, the $16 \Phi_{0}$ input waveforms were reconstructed from the measured data, with an error of less than the flux quantum $\Phi_{0}$.
\end{abstract}

Index Terms-Superconductive sensor, Time division multiplexing, Code division multiplexing, Single flux quantum circuit, Digital SQUID

\section{INTRODUCTION}

$\mathrm{S}$ UPERCONDUCTIVE sensors have extremely high sensitivity and are used in various applications such as biomagnetic measurement using a superconducting quantum interference device (SQUID) magnetometer [1], X-ray detection using a transition edge sensor (TES) [2], and terahertz imaging using a superconducting tunnel junction (STJ) array [3]. The greater the number of sensor channels, the higher is the detection speed and efficiency [4]. However, the number of lines connecting low-temperature and room-temperature environments increases if the multi-channel superconductive sensing system is conventionally built because all sensors are directly controlled and measured by room-temperature

Manuscript received October 9, 2012.

This work was supported by "Promotion of Environmental Improvement for Independence of Young Researchers" under the Special Coordination Funds for Promoting Science and Technology from the Ministry of Education, Culture, Sports, Science and Technology (MEXT) of Japan. The National Institute of Advanced Industrial Science and Technology contributed in part to the circuit fabrication.

K. Aoki, Y. Yamanashi, and N. Yoshikawa are with the Department of Electrical and Computer Engineering, Yokohama National University, Yokohama 240-8501, Japan (corresponding author e-mail: yamanasi@ynu.ac.jp). equipment. In [5], a 1024-channel TES array was built using 4096 lines. However, such a large system is difficult to implement. Furthermore, the large number of lines induces thermal and noise inflow to the sensor array and deteriorates the performance of the sensing systems.

Hybridization of a superconducting sensor array and a superconductive single flux quantum (SFQ) readout circuit that processes and converts output signals from sensors to digital data suitable for data communication from lowtemperature to room-temperature electronics at a high frequency is one of the promising techniques for developing a high-performance multi-channel superconductive sensing system. Thus far, several superconducting sensing systems based on SFQ readout circuits, a digital SQUID magnetometer [6], a digital double relaxation oscillator (DROS) magnetometer [7], and a TES array system using quasi-onejunction SQUID (QOS) for X-ray detection [8] have been proposed and implemented. Because the output signal from each sensor is measured using SFQ circuits that process data at high frequency at the low-temperature stage, a further decrease in the number of lines from the low-temperature stage is possible. Moreover, the performance of the superconductive sensors can be fully utilized because the sensor performance is not limited by the bandwidth of a readout line from the low-temperature stage in this system.

In this study, we have investigated time division multiplexing (TDM), code division multiplexing (CDM), and their circuit structures for a multi-channel superconductive sensing system using SFQ readout circuits. We have estimated the performance of the multi-channel sensing system on the basis of analog circuit simulation. We have implemented and tested a multi-channel digital SQUID magnetometer system using investigated TDM and CDM.

\section{MultiPleXING FOR SFQ CIRCUIT-BASED SENSING SYSTEM}

\section{A. Time Division Multiplexing}

Fig. 1 shows the block diagram of an N-channel superconductive sensing system in TDM using SFQ readout circuits. The output signal from each sensor is converted to SFQ pulses using signal converters $[8,9]$ for data processing. The converted SFQ pulses are input to an $n$-bit SFQ binary counter that counts the number of input SFQ pulses, which correspond to the amplitudes of the output signals. The 


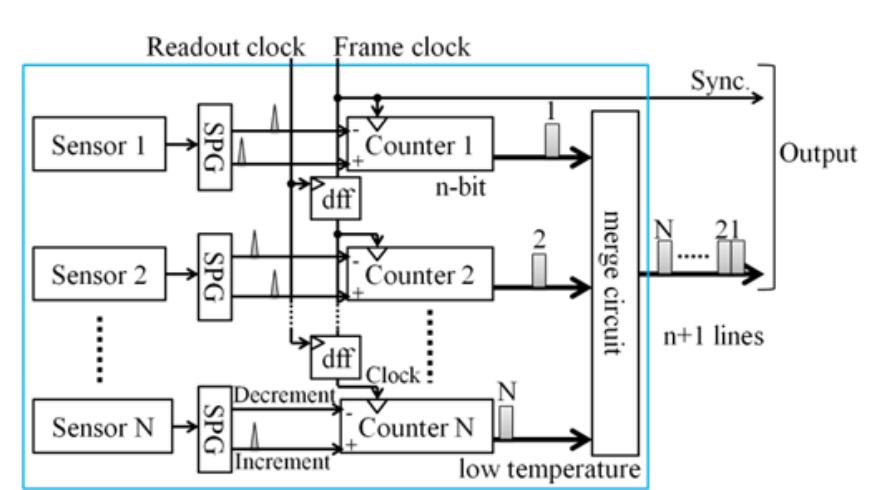

Fig. 1. Block diagram of a time division multiplexing (TDM) circuit for an $\mathrm{N}$-channel sensor array. The SPG corresponds to the SFQ pulse generator. Delay flip-flops (dffs) are inserted for the sequential read out.

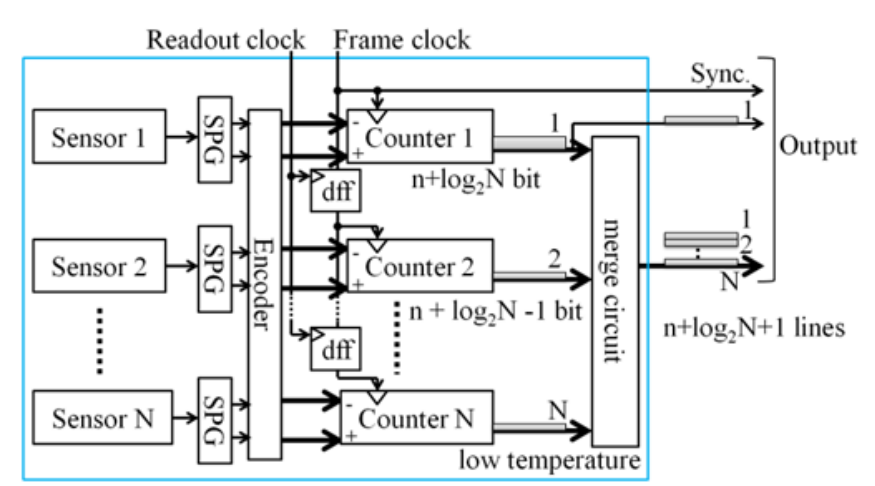

Fig. 2. Block diagram of a code division multiplexing (CDM) circuit for an $\mathrm{N}$-channel sensor array.

required number of bits of the SFQ binary counter, $n$, is determined by the required slew rate of the sensing system. The SFQ counters are read out by readout clock inputs. By reading out the output of the counters sequentially, the output data of each counter are multiplexed in the time domain and can be transmitted to the room-temperature stage using a small number of readout lines.

The TDM circuit can be implemented using simple SFQ binary counters and a merge circuit. Therefore, the circuit structure is relatively simple. The number of lines for input and readout is $n+3$, including output parallel $n$-bit data lines and readout clock input lines for the readout. It should be noted that the number of lines is not proportional to the number of channels $N$. Therefore, TDM is useful for developing multi-channel sensing systems with a small number of lines. However, when the number of channels increases, the effective slew rate of each sensor decreases because counters are read out sequentially and the readout frequency for each counter is proportional to $N$.

\section{B. Code Division Multiplexing}

Fig. 2 shows the block diagram of an N-channel sensing system in CDM using SFQ readout circuits. An encoder in Fig. 2 encodes the output signal from each sensor using a Walsh matrix to make the data from each sensor orthogonal. A Walsh matrix is a square matrix in which the inner product of any two rows is zero. The number of elements in the row vector is $N$. The required number of bits for the counters is greater than

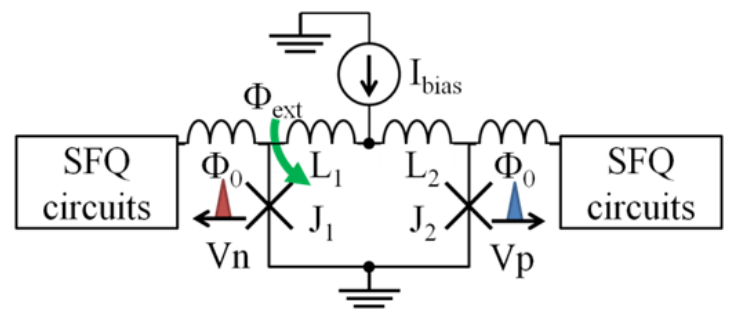

$\overline{(a)}$

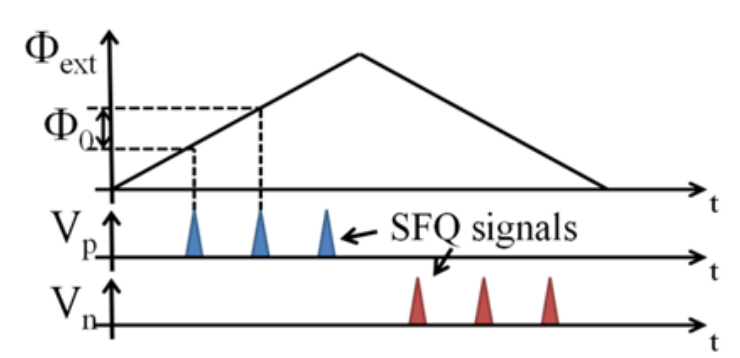

(b)

Fig. 3. (a) Schematic of an asynchronous digital SQUID and (b) operation of the digital SQUID. Whenever the external magnetic flux $\Phi_{\text {ext }}$ increases/decreases by $\Phi_{0}$, an SFQ pulse is output. $\mathrm{I}_{\text {bias }}=0.19 \mathrm{~mA}$, $\mathrm{L}_{1}=50 \mathrm{pH}, \mathrm{L}_{2}=50 \mathrm{pH}, \mathrm{I}_{\mathrm{cl}}=0.1 \mathrm{~mA}, \mathrm{I}_{\mathrm{c} 2}=0.1 \mathrm{~mA}$. $\mathrm{I}_{\mathrm{cl}, 2}$ is a critical current value of $\mathrm{J}_{1,2}$.

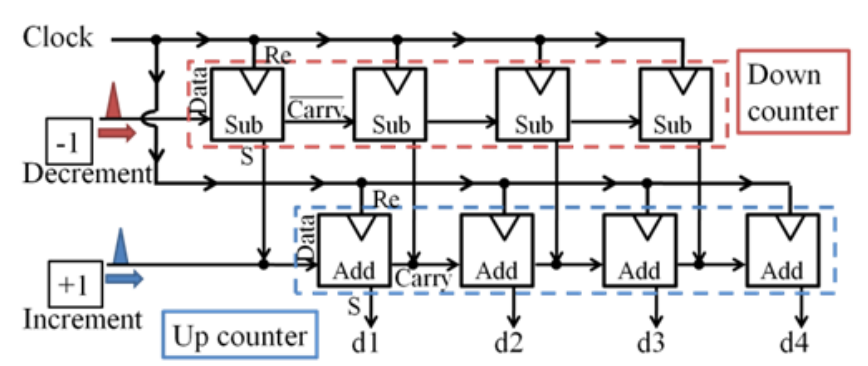

Fig. 4. Block diagram of an 4-bit SFQ up/down counter. "Sub" and "Add" are subtraction and adder cells, respectively. The 4-bit counter can count the input data from -7 to +7 . Output data are read out as parallel data $(\mathrm{d} 1-\mathrm{d} 4)$.

that in the case of TDM by $\log _{2} N$. The encoded data from each sensor can be input to all counters simultaneously, because the encoded data are orthogonal and do not interfere with each other. The data are decoded after readout of the counters by using the inverse Walsh matrix, and the original signals can be reconstructed. The encoded output data from all sensors are input to all counters, and the readouts are synchronized by a readout clock input. Therefore, the effective slew rate of each channel does not decrease with an increase in the number of channels [10]. However, the circuit structure becomes complicated as compared to that of the TDM circuit because an encoder and longer counters are required to implement CDM.

\section{Performance Estimation of Multi-Channel Digital SQUID MAGNETOMETER}

We have designed multi-channel asynchronous digital SQUID magnetometers [11] to estimate the performance of the system by analog circuit simulation. Fig. 3 shows a circuit schematic of the asynchronous digital SQUID and its operation. The external magnetic flux $\Phi_{\text {ext }}$ penetrates a dcSQUID loop and can be measured by SFQ circuits. Whenever $\Phi_{\text {ext }}$ increases by a single flux quantum $\Phi_{0}\left(=2.07 \times 10^{-15} \mathrm{~Wb}\right)$, 


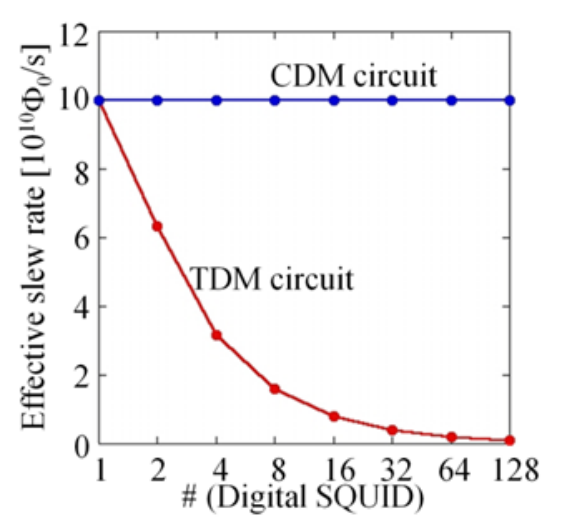

(a)

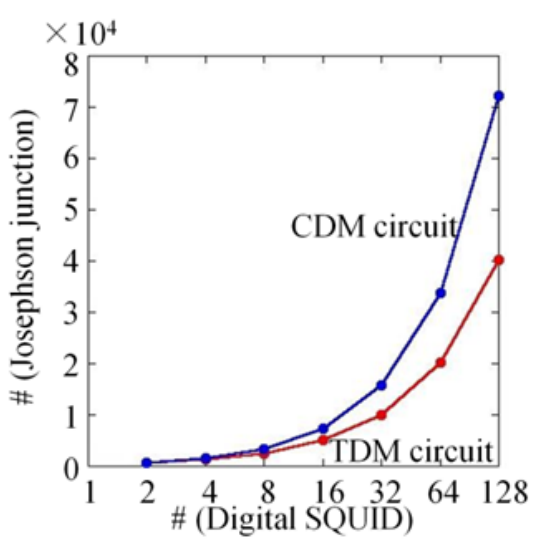

(b)

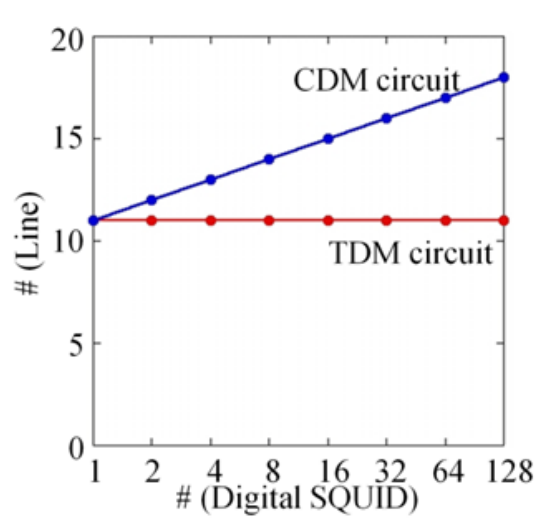

(c)

Fig. 5. Comparison of TDM and CDM circuits. Dependences of (a) the effective slew rate, (b) the circuit scale, and (c) the number of required lines for the multi-channel asynchronous digital SQUID magnetometer on the number of channels for TDM and CDM.

an SFQ pulse is output as the $V_{p}$ signal. The $V_{n}$ signal is output when $\Phi_{\text {ext }}$ decreases by $\Phi_{0}$. The external magnetic flux can be measured with a resolution of $\Phi_{0}$ [11] by counting the number of complementary SFQ outputs. The slew rate of one channel of the asynchronous digital SQUID is estimated to be $1.0 \times$ $10^{11} \Phi_{0} / \mathrm{s}$ [11]. Digital SQUIDs are used in various applications such as a geophysics application [12].

We have implemented TDM and CDM circuits using SFQ up/down binary counters. Fig. 4 shows the block diagram of a 4-bit SFQ up/down counter. The up/down counter comprises adder cells and subtraction cells [13]. The internal state of the counter, which is incremented and decremented by the complementary inputs, is destructively output by inputting the readout clock. We have optimized the circuit parameters to improve the operating frequency. As a result of the optimization, the maximum operational input frequency of the up/down counter reached $167 \mathrm{GHz}$.

Using the results of the circuit design, we have estimated the effective slew rate of each digital SQUID channel, circuit scale, and the required number of lines from the lowtemperature environment for TDM and CDM. In this estimation, the use of 8-bit counters and a $1 \mathrm{GHz}$ readout clock was assumed. Fig. 5 summarizes the dependences of the estimated performance on the number of channels. As discussed in chap. II, the slew rate of the multi-channel digital SQUID in CDM does not depend on the number of channels, whereas the circuit scale of the CDM system becomes greater than that of the TDM system. Because the structure of the CDM circuit is more complicated than that of the TDM circuit, the scale of the CDM circuit is relatively large.

\section{Demonstration of 2-Channel Digital SQUID SYSTEM IN TDM AND CDM}

We have designed and implemented a test circuit for a 2channel asynchronous digital SQUID magnetometer using the SRL (Superconductivity research laboratory) $2.5 \mathrm{kA} / \mathrm{cm}^{2} \mathrm{Nb}$ standard process [14]. Two 4-bit SFQ up/down counters are used in this circuit. Fig. 6 shows the microphotograph of the test circuit. In this test circuit, the multiplexing function can be reconfigured by inputting control signals. The test circuit contains 1649 Josephson junctions.

This test circuit can work as either a TDM or a CDM circuit.
The function can be reconfigured by inputting control signals. To implement CDM for the 2-channel magnetometer the following $2 \times 2$ Walsh matrix is used:

$$
\mathrm{W} \equiv\left(\begin{array}{cc}
1 & 1 \\
1 & -1
\end{array}\right)=2 \mathrm{~W}^{-1}
$$

The encoder in the CDM circuit was designed on the basis of matrix (1). The 2-channel data are encoded according to the following equation:

$$
D=\mathrm{W} S
$$

where $D$ and $S$ are the encoded data that are input to the SFQ counters and the output data from the sensor array. By substituting the following matrixes corresponding to 2-channel data,

$$
D=\left(\begin{array}{ll}
D_{1} & D_{2}
\end{array}\right)
$$

and

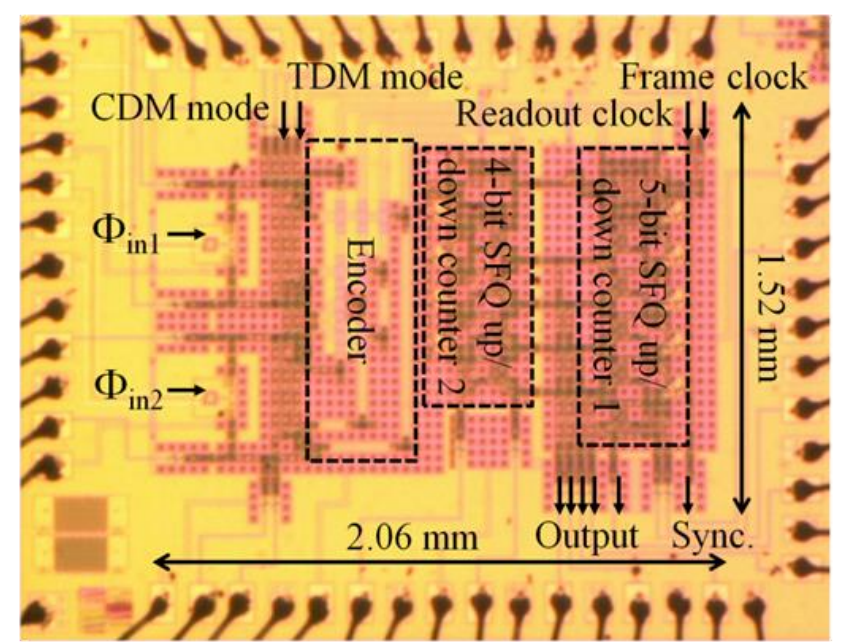

Fig. 6. Microphotograph of a 2-channel digital SQUID system that can work as either a TDM or CDM circuit. TDM and CDM modes are control signals to reconfigure the circuit function. 


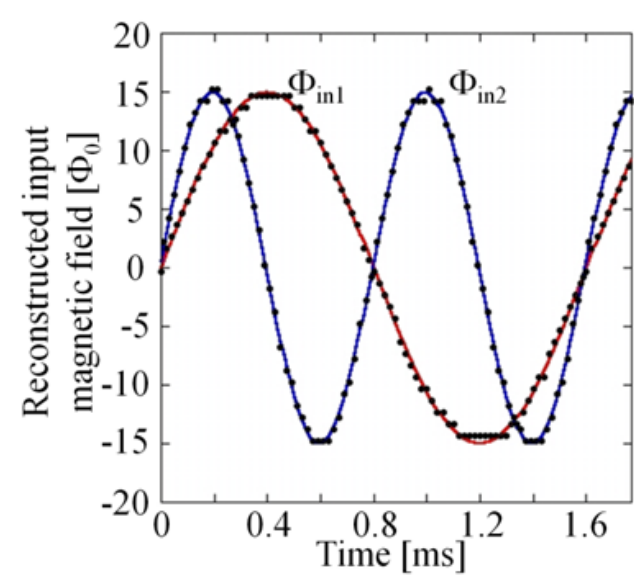

(a)

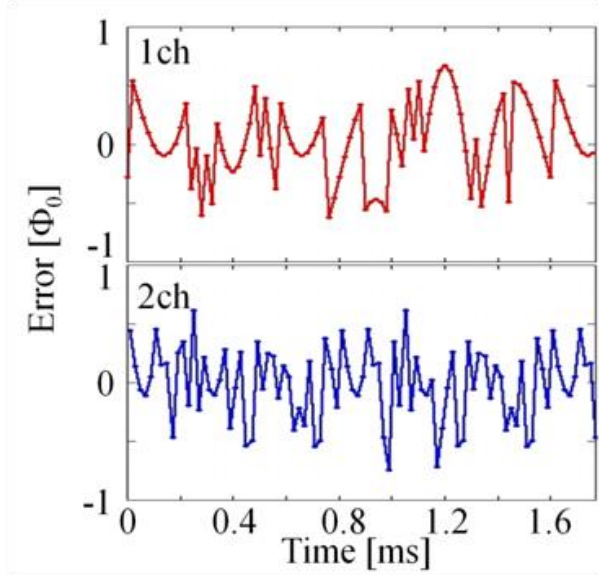

(b)

Fig. 7. (a) Waveform reconstructed from the output data of the TDM circuit and (b) the error. The dots in (a) correspond to the reconstructed waveform, and the lines are input signals.

$$
S=\left(\begin{array}{ll}
S_{1} & S_{2}
\end{array}\right)
$$

the following relations are obtained:

$$
D_{1}=S_{1}+S_{2}
$$

and

$$
D_{2}=S_{1}-S_{2}
$$

where $S_{n}$ is output signal from the $n$-th channel, and $D_{n}$ is the encoded data input to the $n$-th counter. Therefore, the output SFQ signals are added and input to the first counter. The difference of the data from the two sensors is input to the second counter.

We input sinusoidal magnetic signals to each channel and measured the input magnetic flux with the test circuit using both TDM and CDM. Frequencies of the input magnetic flux for channels 1 and 2 were $625 \mathrm{~Hz}$ and $1.25 \mathrm{kHz}$, respectively. The amplitude of the input magnetic flux was $16 \Phi_{0}$. The output data from the two counters were recorded with an oscilloscope. After data acquisition, the recorded output data were processed, and the input waveforms were reconstructed.

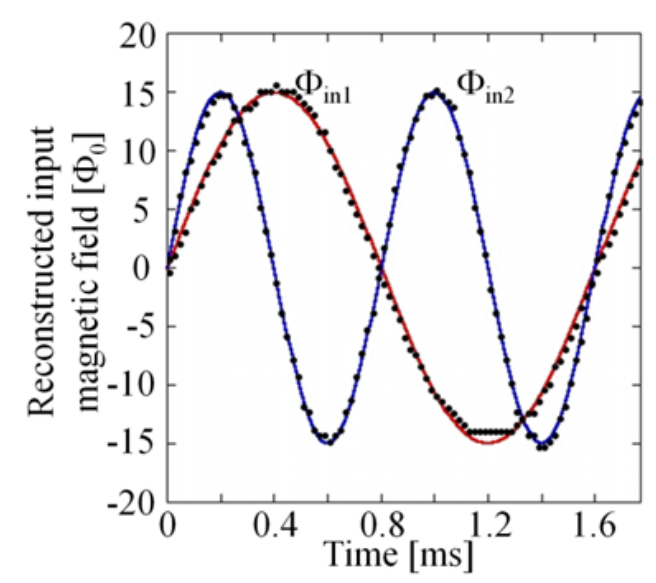

(a)

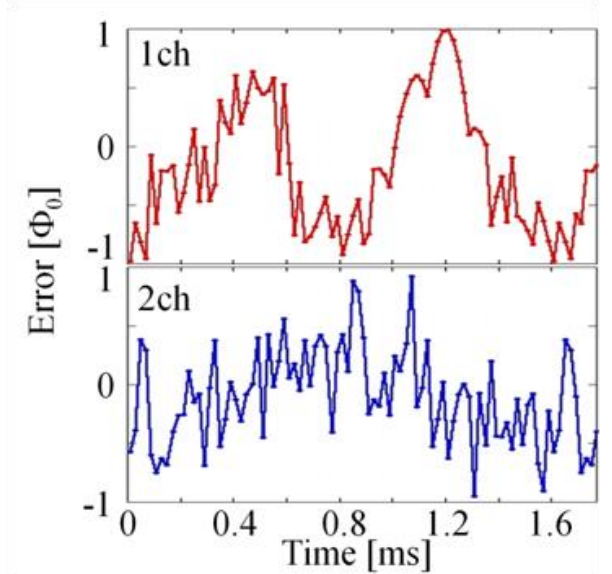

(b)

Fig. 8. (a) Waveform reconstructed from the output data of the CDM circuit and (b) the error. The dots in (a) correspond to the reconstructed waveform, and the lines are input signals.

Figs. 7(a) and 8(a) show the reconstructed waveforms of each channel. It can be confirmed that the input signals can be properly reconstructed from the output data in both TDM and CDM. Figs. 7(b) and 8(b) show the calculated errors of the reconstructed waveforms, which correspond to the difference between the reconstructed data and input waveforms. In both TDM and CDM, the measured error was less than $\Phi_{0}$, as shown in Figs. 7(b) and 8(b).

\section{CONCLUSION}

We have investigated TDM and CDM for multi-channel superconductive sensing systems based on SFQ readout circuits. We have described the advantages and disadvantages of each multiplexing method. We have estimated the performance of a multi-channel sensing system that employs TDM and CDM using circuit design and analog circuit simulation. The TDM circuit is useful for reducing the number of lines for input and readout. A multi-channel sensing system can be built without decreasing the effective slew rate of each sensor by using CDM. We have designed and tested a 2channel digital SQUID magnetometer system that can work in both TDM and CDM modes by using SFQ up/down counters. The input signals were reconstructed with an error less than the magnetic flux quantum. 


\section{REFERENCES}

[1] R. Fagaly, "Superconducting quantum interference device instruments and applications", Rev. Sci. Instrum., 77, 101101, pp. 1-43, Oct. 2006.

[2] D. Prele, M. Piat, E. Breelle, F. Voisin, M. Pairat, Y. Atik, B. Belier, L. Dumoulin, C. Evesque, G. Klisnick, S. Marnieros, F. Pajot, M. Redon, and G. Sou, " Development of Superconducting NbSi TES Array and Associated Readout With SQUIDs and Integrated Circuit Operating at 2 K," IEEE Trans. Appl. Supercond., vol. 19, pp. 501-504, Jun. 2009.

[3] C. Otani, T. Taino, R. Nakano, K. Hoshino, T. Shibuya, H. Myoren, S. Ariyoshi, H. Sato, H. Shimizu, S. Takada, and K. Kawase, "A BroadBand THz Readiation Detector using a Nb-Based Superconducting Tunnel Junction," IEEE Trans. Appl. Supercond., vol. 15, pp. 591-594, Jun. 2005.

[4] J. Zmuidzinas, and P. Richards, "Superconducting Detectors and Mixers for Millimeter and Submillimeter Astrophysics," Proceedings of the IEEE, vol. 92, pp. 1597-1616, Oct. 2004.

[5] M. Niemack, "TOWARDS DARK ENERGY: Design, Development, and Preliminary Data from ACT," Princeton University, Apr. 2008.

[6] T. Reich, T. Ortlepp, and F. Uhlmann, "Digital SQUID Sensor Based on SFQ Technique," IEEE Trans. Appl. Supercond., vol. 15, pp.3-28, Jun. 2005.

[7] H. Myoren, Y. Kimimoto, K. Terui, and T. Taino, "Design of Digital DROS With SFQ Up/Down Counter for Wide Dynamic Operation Range," IEEE Trans. Appl. Supercond., vol. 21, pp.387-390, Jun. 2011.

[8] A. Bozbey, S. Miyajima, H. Akaike, and A. Fujimaki, "Single-FluxQuantum Circuit Based Readout System for Detector Arrays by Using Time to Digital Conversion," IEEE Trans. Appl. Supercond., vol. 19, pp.509-513, Jun. 2009.

[9] H. Terai, S. Miki, and Z. Wang, "Readout Electronics Using SingleFlux-Quantum Circuit Technology for Superconducting Single-Photon Detector Array," IEEE Trans. Appl. Supercond., vol. 19, pp. 350-353, Jun. 2009.

[10] K. Irwin, M. Niemack, J. Beyer, H. Cho, W. Doriese, G. Hilton, C. Reintsema, D. Schmidt, J. Ullom, and L. Vale, "Code-division multiplexing of superconducting transition-edge sensor arrays," Supercond. Sic. Technol., 23, pp. 1-7, Feb. 2010.

[11] Y. Tsuga, Y. Yamanashi, and N. Yoshikawa, "Asynchronous Digital SQUID Magnetometer with an On-Chip Magnetic Feedback for Improvement of Magnetic Resolution," Appl. Supercond. Conf., 4EB-05, Portland, Oct. 2012.

[12] P. Febvre, "Digital SQUID Magnetometer Development for Geophysics Applications Validated in Low-Noise Environment," IEEE/CSC \& EUROPIEN SUPERCOND. NEWS FORUM, no. 5, pp. 1-6, Jul. 2008.

[13] T. Onomi, T. Kondo, and K. Nakajima, "Implementation of High-Speed Single Flux-Quantum Up/Down Counter for the Neural Computation Using Stochastic Logic," IEEE Trans. Appl. Supercond., vol. 19, pp. 626-629, Jun. 2009.

[14] S. Nagasawa, Y. Hashimoto, H. Numata, and S. Tahara, "A 380 ps. 9.5 $\mathrm{mW}$ Josephson 4-kbit RAM operated at a high bit yield," IEEE Trans. Appl. Supercond., vol. 5, pp. 2447-2452, Jun. 1995. 